Journal of Engineering and Applied Sciences 14 (Special Issue 7): 9973-9977, 2019

ISSN: 1816-949X

(C) Medwell Journals, 2019

\title{
Design and Development of Secure Efficient Wireless Communication System based on FHSS
}

\author{
${ }^{1}$ Samir Jasim and ${ }^{2}$ Ahmed M. Alfadhel \\ ${ }^{1}$ Department of Electrical Engineering, University of Babylon, Hillah, Iraq \\ ${ }^{2}$ Department of Communication Techniques, Al-Furat Al-Awsat Technical University, Kufa, Iraq
}

\begin{abstract}
As far as there is a need to increase the efficiency of data transfer, the trials of developments communication systems are appeared. In this study, four modulation techniques and three PN sequence code lengths are studied, to indicate the best performance. The study included BER calculations for each modulation technique or PN code is used in this FHSS system. MATLAB Simulink with run time of 10 is used to simulate each modulation technique. And Additive White Gaussian Noise (AWGN) channel is used as an RF channel. Finally, after we present the simulation results, we deduce which modulation technique is the most suitable for FHSS system and what is the advantage of the longer PN code.
\end{abstract}

$\underline{\text { Key words: FHSS, modulation technique, PN sequence code length, BER, AWGN channel, communication }}$

\section{INTRODUCTION}

One of spread spectrum techniques is Frequency Hopping Spread Spectrum (FHSS). This technique its origins back to the mid-1950s when blades system was made (Scholtz, 1982). Although, FHSS system needs a large number of frequencies but it offers secure transmission, interference rejection, low power transmission, selective addressing and the possibility of multiple access (Dixon, 1994).

A frequency hopping generator is the heart of a FHSS system. In more details, it consists of two main components, the first is PN code generator. It generates a code with a specific lengths such as $8,16,32$ and 64 , so on. In fact, many of military communications systems depends on FHSS, since, the possibility that two FHSS that have different PN codes but occupy the same band is to hop the same frequency at the same time is "more or less randomly" (Dixon, 1994).

The second component is a frequency synthesizer. It works to hop between one frequency and another by depending on the incoming PN sequence code.

Many factors control the efficiency of data transmission in FHSS system such as hopping rate, PN sequence code length, data rate, modulation technique. Two factors are handled here, they are PN code length and modulation technique. Frequency modulation techniques are always used with FHSS, since, the percentage of correct identification is "approximately constant around 99\%” without taking into account C/N value, since, it has no effect when using frequency modulation techniques (Subhi and Jabir, 2008).
Literature review: Establishing a communication link using FHSS technique grants several merits. Hence, this technique is existed in wireless mobile system then in wireless computer networks (Turcotte and Wickert, 1989; Falsafi and Pahlavan, 1995). But it is important to know that FHSS technique is slower than DSSS technique in synchronization process, for this synchronization techniques are proposed to enhance the system (Mason and Felstead, 1991).

By appearance of emerging technologies, engineers found themselves need to make systems that handle enormous amount of data, consume minimum power as possible and with flexible design. Consequently, FHSS technique found its way to be implemented by new methods, pushing the performance of this technique to higher levels. For instance, Wu et al. (2002) implemented a FHSS using a coarse-grained FPGA architecture. While Park et al. (2010) proposed a new algorithm for Adaptive Frequency Hopping (AFH).

FHSS technique found its implementation in many applications. Each of Wu et al. (2002), Park et al. (2010), Jasim et al. (2016) presented a system design to be used with medical technical equipments. Recently, FHSS is used in spectrum sensing for Cognitive Radio (CR) purpose (Saber et al., 2018) and for frequency hopping pattern recognition purpose (Gu and Goodman, 2018).

\section{MATERIALS AND METHODS}

System design: Park et al. (2010) proposed a system design consists of transmitter, frequency hopping generator, transmission channel and receiver. Figure 1 shows a general block diagram of the system. 


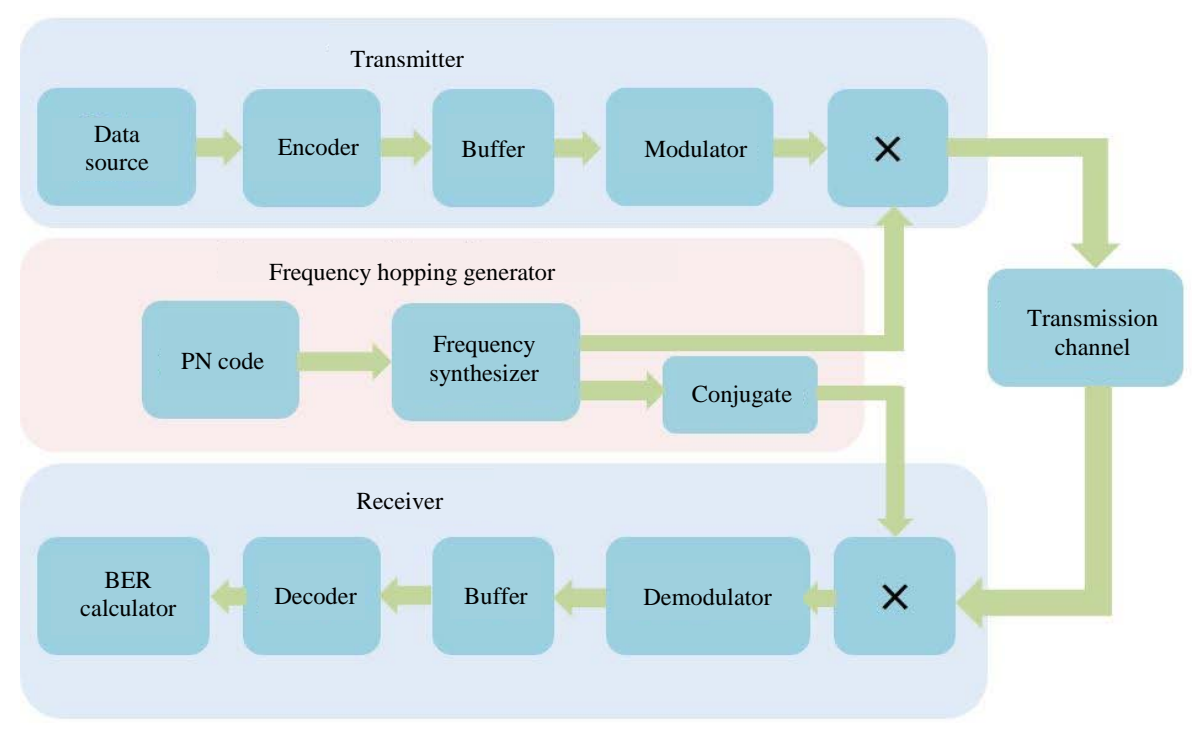

Fig. 1: System design

Transmitter side: Firstly, it consists of Bernoulli Binary Generator block. This block generate a different random data stream at each run. With a specific probability of zeros and ones. Moreover, controlling the sample time in this block will give the required data rate. Bernoulli block is followed by binary cyclic encoder block to correct random errors that occurred during the transmission channel. Where 10/15 encoder rate is used, since, the input message length $(\mathrm{K})$ is 10 and the output codeword length $(\mathrm{N})$ is 15 . Then a buffer will assemble data bits in packets of size 625 bits at rate of $1 \mathrm{Mbps}$. In more details, a data rate of $1 \mathrm{Mbps}$ is achieved as follow:

$$
1 /\left(1.5 \times 10^{-6}\right) \times 15 / 10=1 \mathrm{Mbps}
$$

where, $1.5 \times 10^{-6}$ second is the sample time that is used in Bernoulli block while $15 / 10$ is output to input encoder rate.

After that a modulator block is used to modulate the data bits. In fact, four modulation techniques will be implemented in this design which they are CPM (Continuous Phase Modulation), CPFSK (Continuous Phase Frequency Shift Keying), MSK (Minimum Shift Keying) and GMSK (Gaussian Minimum Shift Keying).

Channel: An Additive White Gaussian Noise (AWGN) channel is used with the system design. The channel is inserted after the modulated signal is spread by a high frequency coming from the hopping generator.

Signal to Noise ratio $\left(E_{b} / N_{o}\right)$ mode is used with input signal power, referenced to $1 \Omega$ is $0.208 \mathrm{~W}$ and symbol period is $10^{-6}$ sec.
Frequency hopping generator: The main purpose of this part of the design is to select a hopping frequency from a set of frequencies. In practical situation the hopping generator is existed in transmitter side and receiver side. Furthermore, a synchronization is required between both generators in order todespread (correlate) the received signal correctly.

A Linear Feedback Shift Register (LFSR) with maximal length of 63 is used with $1 / 9600$ sec sample time and 6 samples per frame. These parameters give a hopping frequency of $1600 \mathrm{hop} / \mathrm{sec}$.

Then a 64 FSK modulator is used as a frequency synthesizer. At each time 6 samples arrived from the LFSR, then a frequency hop is selected. One more thing, a separation frequency of $1 \mathrm{MHz}$ is used between one hop and another.

Receiver side: Firstly, a multiplier is used to multiply each of the AWGN channel output signal and the conjugate of the hopping frequency signal in order to correlate (despread) the spread signal. Then a demodulator block is used, this block is required to demodulate the despread signal to a baseband signal. After that data packets are disassembled by buffer block. Finally, to get the original transmitted data signal we have to decode the disassembled signal by using binary cyclic decoder block. One more block is used at the receiver, for error rate calculations which it is BER block. The total design is carried out by MATLAB Simulink. Figure 2 shows the complete design of the system. 


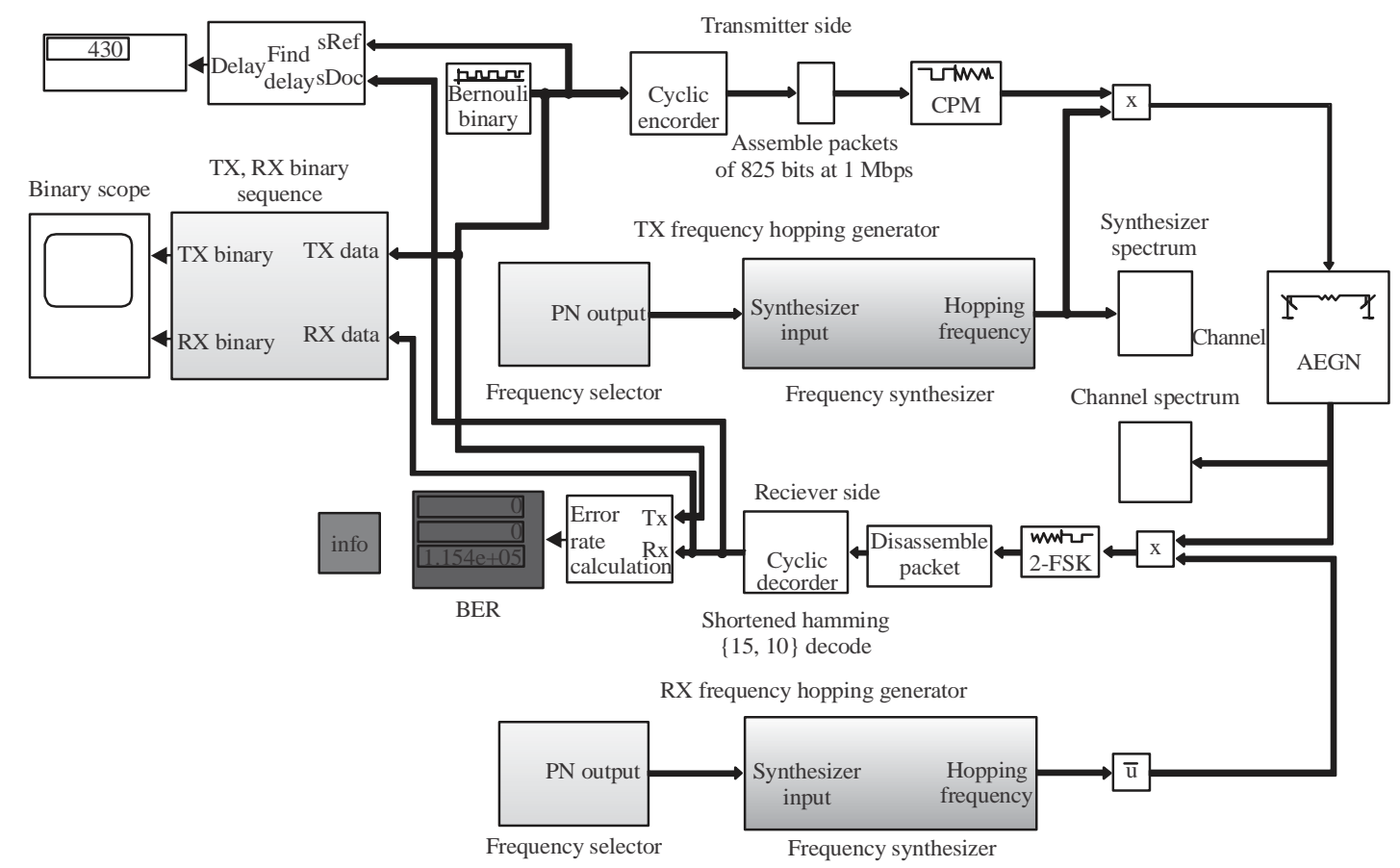

Fig. 2: Complete system design

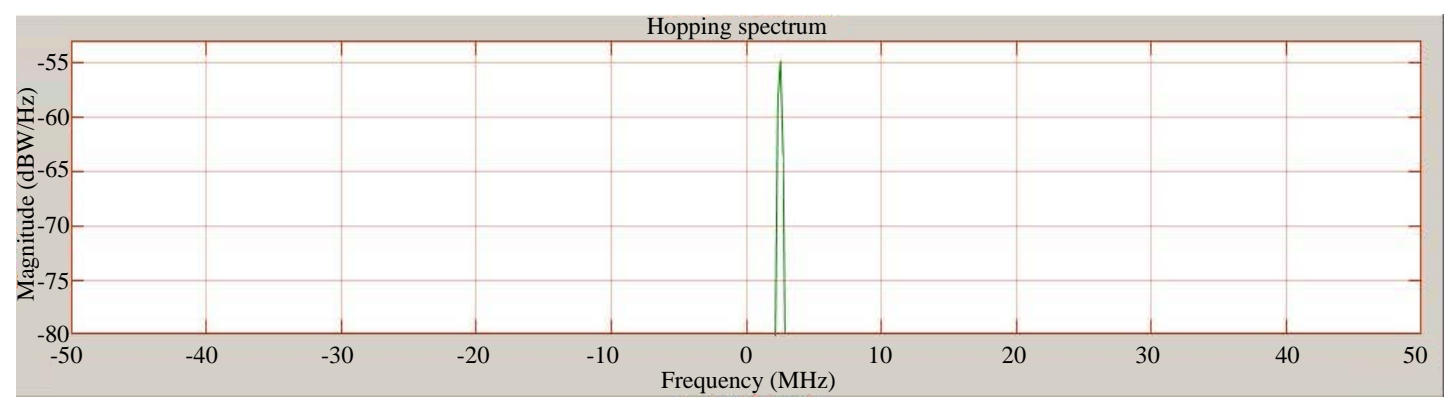

Fig. 3: Frequency synthesizer output

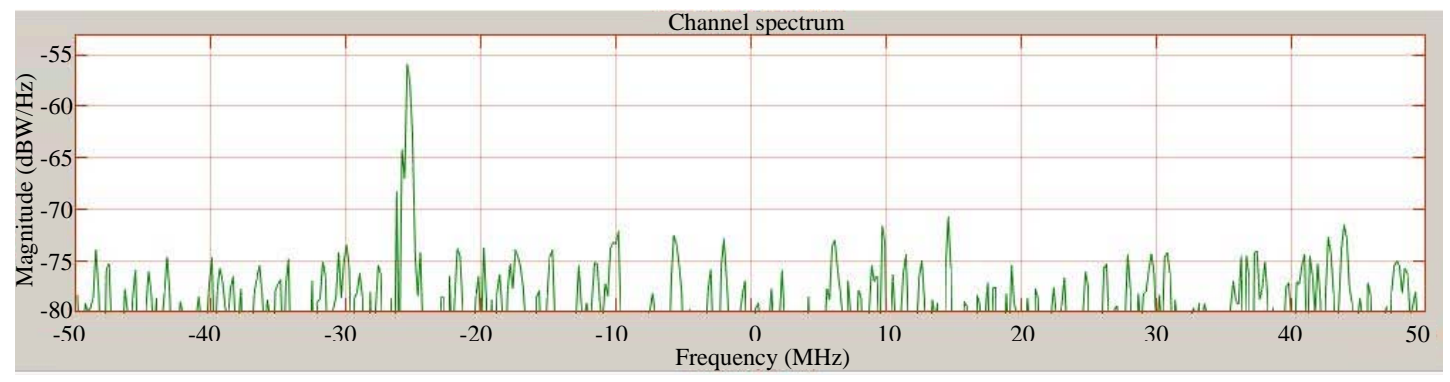

Fig. 4: AWGN channel output

\section{RESULTS AND DISCUSSION}

It is more appropriate to view the hop spectrum and channel spectrum before listing the main results sections for this Fig. 3 and 4 show that, respectively.
In Fig. 3 the spectral component is pointing to $2 \mathrm{MHz}$, however, a $2 \mathrm{MHz}$ here stands for $34 \mathrm{MHz}$, the actual component, since, the analyzer split the 64 hopping frequencies into two halves by set 32 frequencies in the positive side and the same 


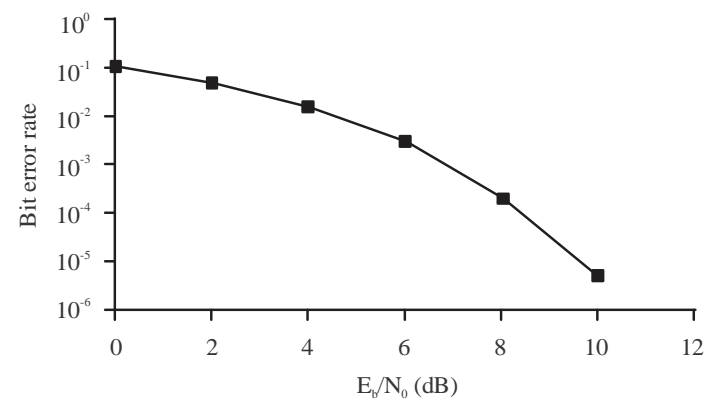

Fig. 5: BER versus $E_{b} / N_{o}$ in FHSS system with CPM modulated signal

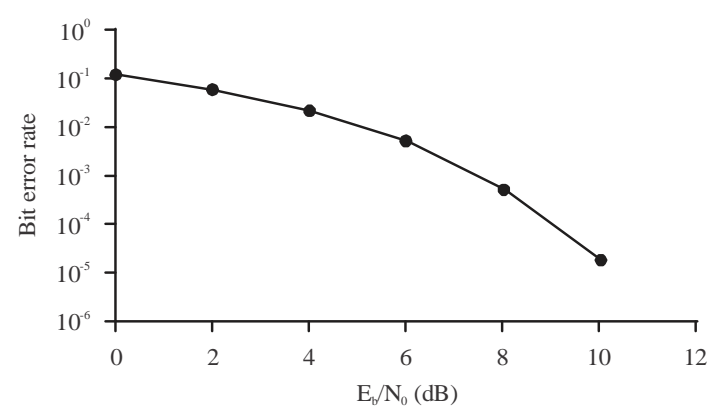

Fig. 6: $B E R$ versus $E_{b} / N_{o}$ in FHSS system with CPFSK modulated signal

in the negative side. While in Fig. 4 the spectral component is pointing to $-26 \mathrm{MHz}$ which stands for $6 \mathrm{MHz}$.

The rest of results are divided into five sections. In the first four sections, the transmission and reception is carried out using the four modulation techniques, one by one. In the meanwhile, BER is calculated over a set of $E_{b} / N_{o}$ values. While final section is dedicated to test three PN sequence code lengths with the modulation technique that gave best BER calculations.

Section 1; CPM: CPM technique is used as a modulator with Gaussian pulse shape while 2-FSK demodulator block is used at receiver side, since, 2-FSK demodulator gave a better performance than CPM demodulator. Figure 5 shows BER versus set values of $E_{b} / N_{o}$ in FHSS system with CPM modulated signal.

Section 2; CPFSK: CPFSK technique is used as a modulator, also 2-FSK demodulator is used in receiver side, since, 2-FSK demodulator gave a better performance.

Here, BER calculations showed a significant rise in comparison with CPM modulation technique. Figure 6 shows BER versus set values of $E_{b} / N_{o}$ in FHSS system with CPFSK modulated signal.

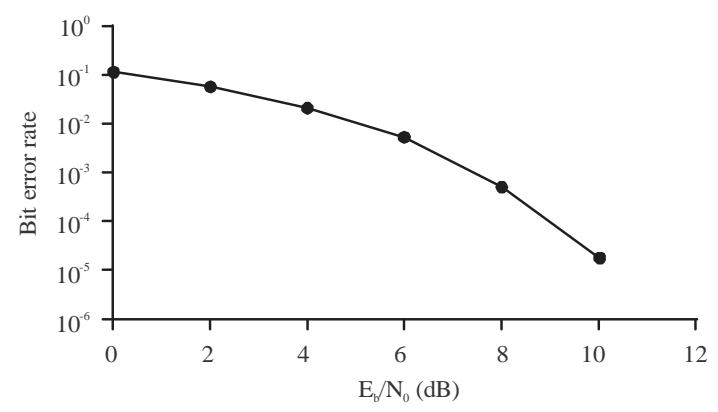

Fig. 7: BER versus $E_{b} / N_{o}$ in FHSS system with MSK modulated signal

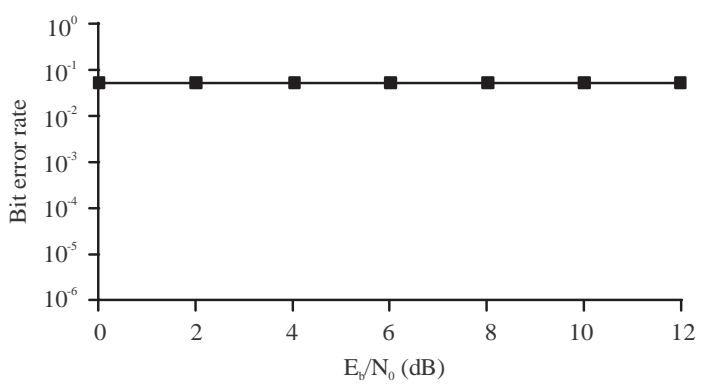

Fig. 8: BER versus $E_{b} / N_{o}$ in FHSS system with GMSK modulated signal

Section 3; MSK: In this case MSK modulator block is used in the transmitter side and also 2-FSK demodulator block is used in the receiver side for same previous reason.

Back to our point of study, BER calculations in this case showed a similar behavior with previous case. In fact, there is no difference between MSK and CPFSK in BER calculations as shown in Fig. 7.

Section 4; GMSK: In this case GMSK modulator block is used in the transmitter side while GMSK demodulator block is used in the receiver side.

However, BER calculations showed a steady behavior over the selected set of $E_{b} / N_{o}$ values. In fact, BER values were equal to 0.05 . As shown in Fig. 8. To sum up, Fig. 9 shows BER behavior of the four modulation techniques together.

It is clear that CPM modulation technique gave the best BER in this FHSS system design, in comparison to the other three types.

Section 5; 16, 32, 64 PN code lengths: In this time, the PN code length is altered, while CPM technique is remained unchanged in each case. As in previous sections, a set of $E_{b} / N_{o}$ values are taken to measure BER each time.

In fact, a simulation time of a period of 50 shows almost same behavior for the three lengths. Figure 10 shows BER measurements for them. 


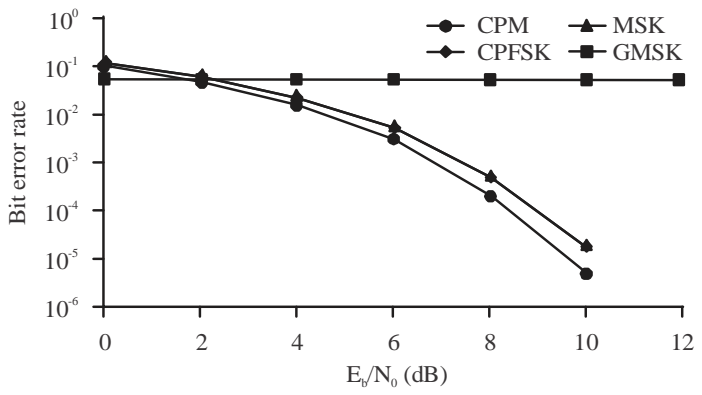

Fig. 9: BER versus $E_{b} / N_{0}$ in FHSS system of four modulated signals

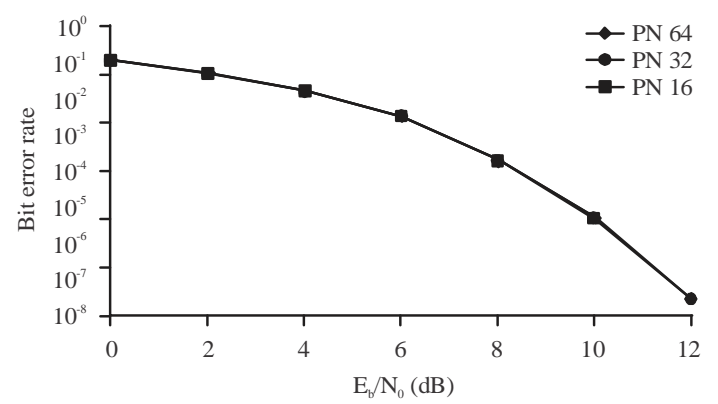

Fig. 10: BER versus $E_{b} / N_{o}$ in FHSS system for three PN code lengths

\section{CONCLUSION}

In this FHSS system, we have tested four modulation techniques and three PN sequence code lengths. The implementation results showed that lowest BER can be achieved using CPM technique.

On the other side, the test results of PN code lengths, showed that they don't affect BER value. This leads to a conclusion about PN length, that the longer PN sequence code will have the lower probability to hop the same frequency at the same time with another PN code located in the same band.

Finally, for further work in future, it is possible to investigate the possibility of increasing data rate of FHSS system. And this research is adhesive with increasing hopping rate of FHSS system.

\section{ACKNOWLEDGEMENTS}

The researchers would like to thank Hamzah Sabr, $\mathrm{PhD}$ candidate student for his valuable discussions and his continuous support for this research.

\section{REFERENCES}

Dixon, R.C., 1994. Spread Spectrum Systems: With Commercial Applications. 3rd Edn., Wiley, Hoboken, New Jersey, USA., ISBN: 978-0-47159342-3, Pages: 592.
Falsafi, A. and K. Pahlavan, 1995. A comparison between the performance of FHSS and DSSS for wireless LANs using a 3D ray tracing program. Proceedings of the 1995 IEEE 45th Vehicular Technology Conference on Countdown to the Wireless Twenty-First Century Vol. 2, July 25-28, 1995, IEEE, Chicago, IL, USA., pp: 569-573.

Gu, Y. and N.A. Goodman, 2018. Information-theoretic compressive measurement for frequency hopping pattern recognition. Proceedings of the 2018 IEEE International Radar Conference (Radar Conf 18), April 23-27, 2018, IEEE, Oklahoma City, OK, USA., pp: 1445-1449.

Jasim, S., A.T. Jaiad and H. Sabr, 2016. Security enhancement of wireless systems by using frequency Hopping Technique. Int. J. Comput. Sci. Mob. Appl., 4: 7-14.

Mason, L.J. and E.B. Felstead, 1991. An estimation method for fine-time synchronization of $\mathrm{FH}$ systems using the early-late filter technique. Proceedings of the MILCOM91-Conference Record, November 4-7, 1991, IEEE, McLean, VA, USA., pp: 1059-1063.

Park, K.J., T.R. Park, C.D. Schmitz and L. Sha, 2010. Design of robust adaptive frequency hopping for wireless medical telemetry systems. IET. Commun., 4: 178-191.

Saber, M., H.K. Aroussi, A. El Rharras and R. Saadane, 2018. Raspberry pi and RTL-SDR for spectrum sensing based on FM real signals. Proceedings of the 20186th International Conference on Multimedia Computing and Systems (ICMCS 18), May 10-12, 2018, IEEE, Rabat, Morocco, pp: 1-6.

Scholtz, R.A., 1982. The origins of spreadspectrum communications. IEEE Trans. Commun., 30: 822-854.

Subhi, W. and S. Jabir, 2008. Modulation and frequency estimation of FHSS signal in the presence of interference environments. Proceedings of the 1st International Regional Conference of Engineering Science, November 5-6, 2008, Nahrain University, Baghdad, Iraq, pp: 1-19.

Turcotte, R.L. and M.A. Wickert, 1989. Exact probability of error analysis for FHSS/CDMA communications in the presence of single term Rician fading. Proceedings of the IEEE Military Communications Conference on Bridging the Gap, Interoperability, Survivability, Security, October 15-18, 1989, IEEE, Boston, MA., USA., pp: 134-138.

Wu, W., S.S. Chin and S. Hong, 2002. A coarse-grained FPGA architecture for reconfigurable baseband modulator/demodulator. Proceedings of the 36th International Conference on Record of the Asilomar Signals, Systems and Computers, November 3-6, 2002, IEEE, Pacific Grove, California, USA., pp: 1613-1618. 